\title{
DENIS Observations of Multibeam Galaxies in the Zone of Avoidance
}

\author{
A. Schröder ${ }^{1,2}$, R. C. Kraan-Korteweg ${ }^{3}$ and G. A. Mamon ${ }^{4,5}$ \\ 1 Australia Telescope National Facility, CSIRO, PO Box 76, Epping, NSW 2121, Australia \\ aschroed@atnf.csiro.au \\ 2 Institute of Astronomy, NCU, Chung-Li, 32054 Taiwan \\ 3 Depto. de Astronomía, Univ. de Guanajuato, Ap. P. 144, Guanajuato, \\ GTO 36000, Mexico \\ kraan@astro.ugto.mx \\ 4 IAP, 98bis Blvd Arago, 75014 Paris, France \\ gam@iap.fr \\ 5 DAEC, Observatoire de Paris-Meudon, 92195 Meudon, France \\ Received 1998 November 9, accepted 1999 February 2
}

\begin{abstract}
Roughly $25 \%$ of the optical extragalactic sky is obscured by the dust and stars of our Milky Way. Dynamically important structures might still lie hidden in this zone. Various surveys are presently being employed to uncover the galaxy distribution in the Zone of Avoidance (ZOA), but all suffer from (different) limitations and selection effects. We illustrate the promise of using a multi-wavelength approach for extragalactic large-scale studies behind the ZOA, i.e. a combination of three surveys, optical, systematic blind $\mathrm{H}_{\mathrm{I}}$ and near-infrared (NIR), which will allow the mapping of the peculiar velocity field in the ZOA through the NIR Tully-Fisher relation. In particular, we present here the results of cross-identifying Hi-detected galaxies with the DENIS NIR survey, and the use of NIR colours to determine foreground extinctions.
\end{abstract}

Keywords: galaxies: distances — galaxies: photometry — large scale structure of the universe

\section{Introduction}

Understanding the origin of the peculiar velocity of the Local Group and the dipole in the Cosmic Microwave Background is one of the major goals of the study of large-scale structures. Theoretical reconstruction methods, however, still suffer from large interpolation uncertainties across the Zone of Avoidance (ZOA), which extends over about $25 \%$ of the optically visible extragalactic sky. Dynamically important structures might still lie hidden in this zone, such as the recently discovered nearby Dwingeloo galaxy (Kraan-Korteweg et al. 1994) and the rich massive cluster Abell 3627 (Kraan-Korteweg et al. 1996). Important large-scale structures, e.g. the Supergalactic Plane and other filaments and wall-like structures, seem to continue across this zone. Results from various theoretical approaches still vary strongly, however, in predicting clusters and voids even in the nearby universe (Sigad et al. 1998). This is mainly due to differences in the theoretical reconstruction methods and the adopted parameters, the different galaxy samples to which they are applied, and the selected interpolation mechanisms to bridge the scarcity of data in the ZOA. A more complete knowledge of the distribution of galaxies in redshift space as well as in distance space will improve the reconstructed galaxy density fields and reduce the diversity in the theoretical predictions.

Various approaches are presently being employed to uncover the galaxy distribution in the ZOA (cf. Kraan-Korteweg \& Woudt 1999, this issue p. 53): deep optical searches, NIR surveys (DENIS and 2MASS), far-infrared (FIR) surveys (e.g. IRAS), and blind Hi searches. All methods produce new results, but all suffer from limitations and selection effects. The combination of data from an optical galaxy search, an NIR survey and a systematic blind Hi survey will allow us to examine the large-scale structures behind the southern Milky Way and the peculiar velocity field associated with them. Redshift independent distance estimates can be obtained via the NIR Tully-Fisher relation.

A deep optical survey in the southern ZOA is being conducted by one of us (Kraan-Korteweg \& Woudt 1994; Kraan-Korteweg et al. 1995, 1996, and references therein). In this region $\left(265^{\circ} \lesssim \ell \lesssim 340^{\circ}\right.$, $|b| \lesssim 10^{\circ}$ ), over 11000 previously unknown galaxies above a diameter limit of $D=0.2$ and with $B \lesssim 19{ }^{\mathrm{m}} 0-19 \mathrm{~m}^{\mathrm{m}} 5$ have been identified, next to the previously known $\sim 300$ Lauberts galaxies with 
$D=1^{\prime}$ (Lauberts 1982). Galaxies can be identified visually for $A_{B} \lesssim 5^{\mathrm{m}}$ (or typically down to about $|b| \simeq 5^{\circ}$ depending on surface brightness and Galactic longitude).

The Multibeam (MB) survey of the ZOA, a systematic blind Hi survey (Staveley-Smith 1997), will penetrate even the deepest extinction layers and trace gas-rich spirals out to redshifts of $12000 \mathrm{~km}$ $\mathrm{s}^{-1}$. The survey is currently being conducted with the Multibeam Receiver (13 beams in the focal plane array) at the $64 \mathrm{~m}$ Parkes telescope and will cover the whole southern ZOA $\left(213^{\circ} \lesssim \ell \lesssim 33^{\circ} ;|b| \lesssim 5^{\circ}\right)$ with a $5 \sigma$ detection limit of $10 \mathrm{mJy}$. Hardly any of the 1500 predicted detections are expected to have an optical counterpart, but at these latitudes many might be visible in the NIR. This new prospect becomes feasible with the currently ongoing NIR surveys such as DENIS (DEep NIR southern sky Survey; Epchtein 1997; Epchtein et al. 1997) and 2MASS (Skrutskie et al. 1997). First results from the DENIS survey, which is expected to cover the entire southern sky by the year 2001, are discussed in the following sections.

\section{The DENIS Survey}

Observations in the NIR can provide important complementary data to other surveys. NIR surveys will

- be sensitive to early-type galaxies - tracers of massive groups and clusters - which are missed in IRAS and Hi surveys,

- have less confusion with Galactic objects compared to FIR surveys,

- be less affected by absorption than optical surveys. Furthermore, because recent star formation contributes only little to the flux at this wavelength, the NIR gives a better estimation of the stellar mass content of galaxies and is hence ideally suited for the application of the Tully-Fisher relation.

The DENIS survey has currently imaged $40 \%$ of the southern sky in the $I(0.8 \mu), J(1.25 \mu)$ and $K_{s}(2 \cdot 15 \mu)$ passbands with a resolution of $1^{\prime \prime}$ in $I_{c}$ and $3^{\prime \prime}$ in $J$ and $K_{s}$. In a pilot study, we have assessed the performance of the DENIS survey at low Galactic latitudes (Schröder et al. 1997, hereafter Paper I; Kraan-Korteweg et al. 1998, hereafter Paper II). We (a) tested the potential of the DENIS survey to detect galaxies where optical and FIR surveys fail, i.e. at high foreground extinctions and in crowded regions, (b) established that the NIR colours of galaxies lead to values for the foreground extinction, (c) determined preliminary $I_{c}^{0}, J^{0}$ and $K_{s}^{0}$ galaxy luminosity functions in the rich cluster A3627, and (d) cross-identified highly obscured galaxies detected in a blind Hi search at $|b|<5^{\circ}$.

Number counts of galaxies are lower in the ZOA due to the high foreground extinction, but the effect depends on wavelength. Interpolating from
Cardelli, Clayton \& Mathis (1989), the extinctions in the NIR passbands are $A_{I_{c}}=0.45, A_{J}=0.21$ and $A_{K_{s}}=0.09$ for $A_{B}=1.0$, hence the decrease in number counts as a function of extinction is considerably slower in the NIR than in the optical. Figure 1 shows the predicted surface number density of galaxies for DENIS (Mamon et al. 1998) and for $B<19$, as a function of Galactic foreground extinction (cf. Paper II).

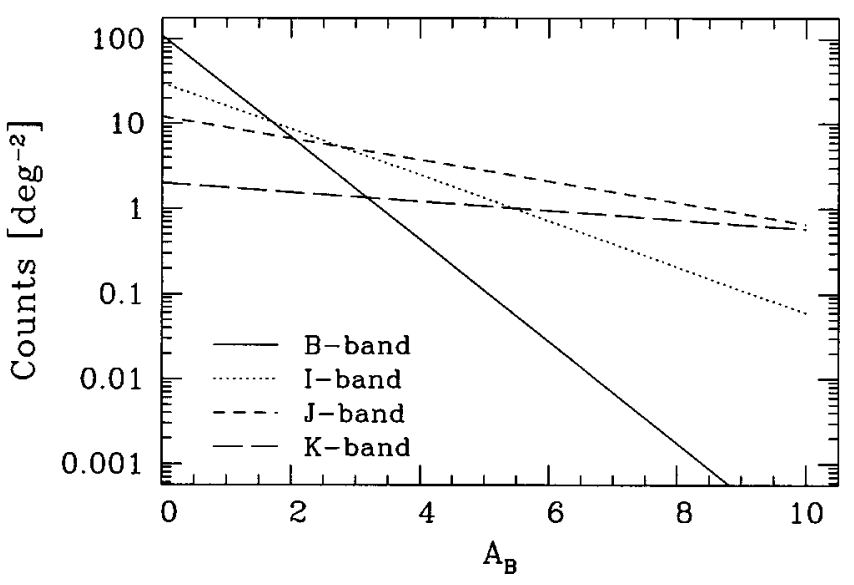

Figure 1-Predicted galaxy counts in $B, I_{c}, J$ and $K_{s}$ as a function of absorption in $B$, for highly complete and reliable DENIS galaxy samples and a $B_{J} \leq 19^{\mathrm{m}}$ optical sample.

The NIR becomes notably more efficient at $A_{B} \gtrsim 2-3^{\mathrm{m}}$. The $J$-band is the most efficient passband to find galaxies at intermediate extinctions, but at $A_{B} \simeq 10^{\mathrm{m}} K_{s}$ becomes superior to $J$. Even at $A_{B}>10^{\mathrm{m}}$ we can expect to find galaxies in $J$ and $K_{s}$. A new air conditioning system for the $K_{s}$-band camera installed in April 1997 has decreased its instrumental background by 0.7 , which makes the $K_{s}$-band now more comparable to $J$. These are very rough predictions and do not take into account any dependence on morphological type, surface brightness, orientation and crowding, which may lower the number of actually detectable galaxies (Mamon 1994).

\section{Cross-identification of $\mathrm{HI}$-detected Galaxies on DENIS Images}

Figure 2 displays the distribution of MB galaxies in the ZOA (large circles). Dots indicate galaxies found in the optical B-band search with a diameter limit of $D=0.2$ (Woudt et al. 1998). Contours indicate extinction levels determined from the DIRBE maps (Schlegel, Finkbeiner \& Davis 1998), which are scaled to independent extinction determinations from $\mathrm{Mg}_{2}$-indices in this region (Woudt 1998). The thick contour corresponds to $A_{B}=2$. 75 ; this is the completeness limit for galaxies with an extinctioncorrected diameter of $D^{0}=1.3$ in the deep optical ZOA galaxy catalogue. The other contours indicate 


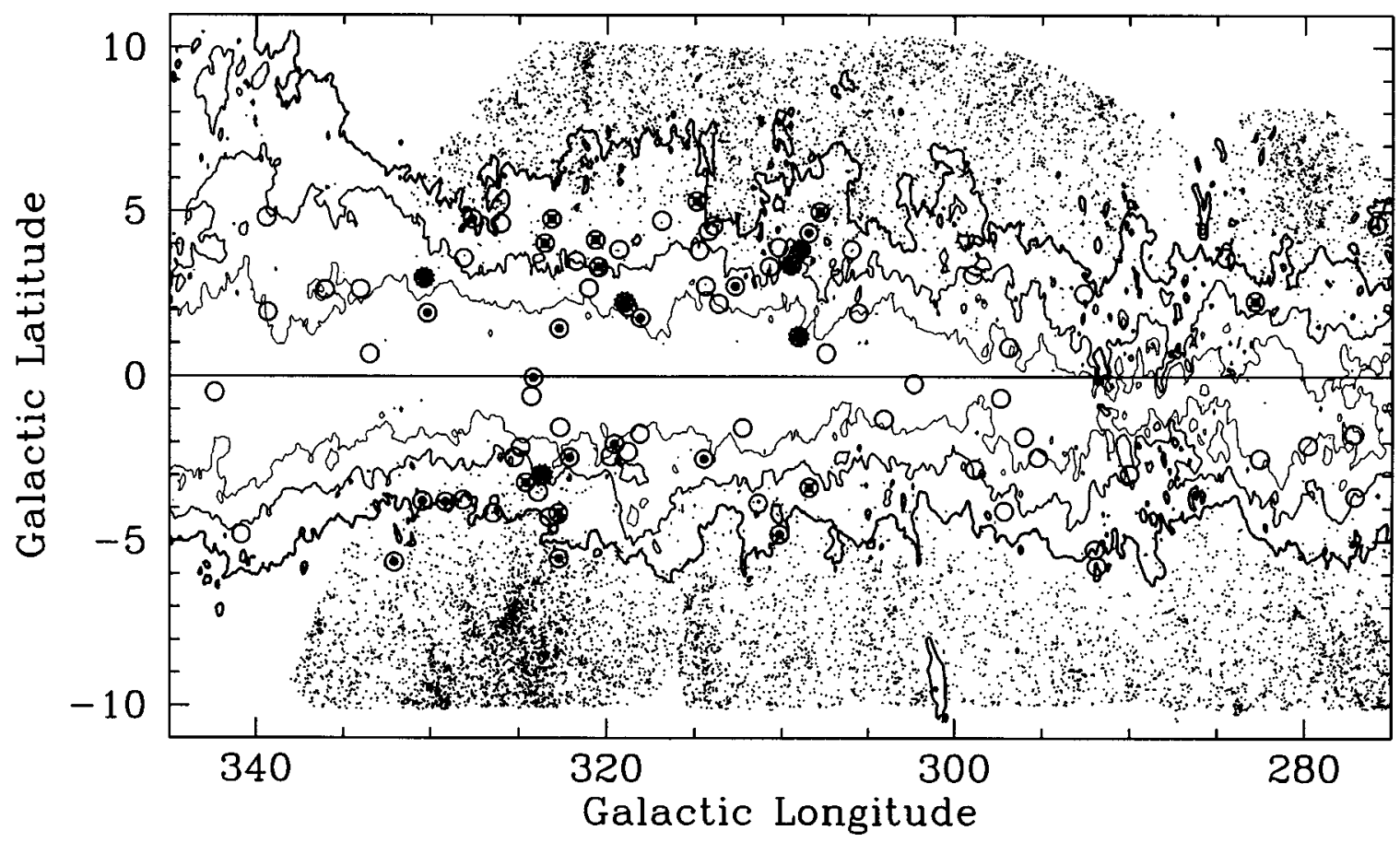

Figure 2- Galaxy distribution in part of the southern ZOA with galaxies found in an optical search (small dots) and galaxies detected with the shallow MB survey (large circles). The superimposed contours represent absorption levels of $A_{B}=2$. 75 (thick line), $5.0,10{ }^{\mathrm{m}} 0$ (see text for details). MB galaxies for which DENIS data are available are marked with a large dot, those re-identified on DENIS images with a cross, and those without an optical counterpart with a star.

$A_{B}=5^{\mathrm{m}}$ and $10^{\mathrm{m}}$, where the Milky Way is opaque in the optical.

For 24 of the galaxies detected in the shallow MB-ZOA survey (cf. Henning et al. 1999 this issue p. 35), DENIS survey images $\left(12^{\prime} \times 12^{\prime}\right)$ covering the full positional uncertainty region $\left(\sim 4^{\prime} \times \sim 4^{\prime}\right)$ are currently available. They are displayed as large dots (including eight detections for which only partial coverage of the Hi positional uncertainty region was available on existing DENIS images). For 16 out of the 24 a clear counterpart could be identified (crosses); of these, six are invisible in the $B$-band due to large foreground extinctions (stars). For four out of the $24 \mathrm{MB}-\mathrm{ZOA}$ galaxies the cross-identifications are uncertain, and for the remaining four galaxies no counterparts could be identified. These galaxies either lie behind a very thick extinction layer (e.g. one galaxy at $b \simeq 0^{\circ}$ with $A_{B} \sim 70^{\mathrm{m}}$ ), or they are late-type spirals or irregular galaxies of very low surface brightness and hence below the magnitude limits of the DENIS survey. The Hi survey, however, is not affected by the foreground extinction, and spiral galaxies can be found in the NIR at lower Galactic latitudes and higher foreground extinction levels than in the optical.

In Figure 3, DENIS images of five MB galaxies are presented. The $I_{c}$-band is shown in the upper panel, the $J$-band in the middle and the $K_{s}$-band in the bottom panel. From left to right the foreground extinction increases. The first galaxy is a nearby $\left(v=1445 \mathrm{~km} \mathrm{~s}^{-1}\right)$ Lauberts galaxy (ESO223-G12) at $b=+4.8$ and $A_{B}=3.9$. It is prominent in all three NIR passbands (note the larger image scale for this galaxy, i.e. $3 ! 3$ instead of $1 ! 7$ ). The second galaxy, also detected with IRAS, at $\left(b, A_{B}\right)=\left(-3.4,4 .^{\mathrm{m}} 4\right)$ is slightly more distant $\left(v=2789 \mathrm{~km} \mathrm{~s}^{-1}\right)$. This galaxy has also been identified in $B$ and is quite distinct in the NIR. The third and fourth galaxies at $\left(b, A_{B}\right) \simeq(3.4,4.8)$ and $(2.3,8.4)$, respectively, have no optical counterparts. Here, the extinction effects are quite obvious: while both have a similar appearance in the $J$-band, the third is very faint in $K_{s}$ and the fourth very faint in $I_{c}$. The fifth example has been detected at the highest extinction so far: $\left(b, A_{B}, v\right) \simeq(+1.2,11.8), 3963 \mathrm{~km} \mathrm{~s}^{-1}$. It is fully obscured in the $I_{c}$-band and barely visible in the $J$-band.

Cross-identifications of galaxies detected in the MB survey are not always unambiguous. The positional accuracy for galaxies detected in the blind Hi search is of the order of $\sim 4^{\prime}$. Sometimes more than one possible counterpart lies within this area as illustrated in Figure 4. The position of this MB galaxy is indicated by a white rectangle on the DENIS $I_{c}$-band image; the foreground extinction is $A_{B}=4 \cdot 0$. Four galaxies can be seen in its vicinity: an edge-on spiral with a small companion (right and above the rectangle), an early-type spiral (left) and a late-type spiral with low surface brightness (left and above the early-type spiral). Although the last seems the most likely candidate given the Hi parameters $\left(v=2903 \mathrm{~km} \mathrm{~s}^{-1}, w=177 \mathrm{~km} \mathrm{~s}^{-1}, I=13 \cdot 1 \mathrm{Jy} \mathrm{km}\right.$ 

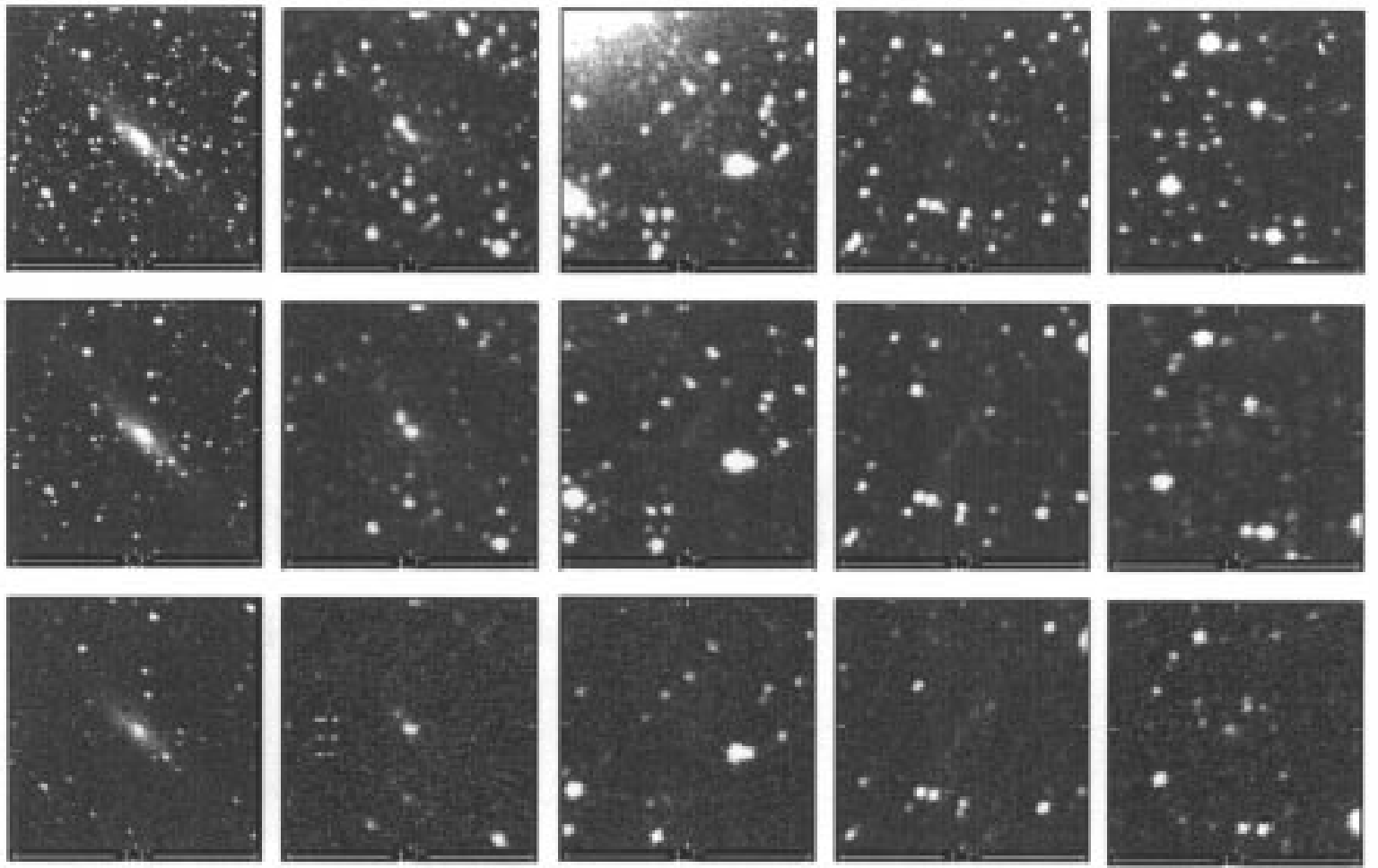

Figure 3-DENIS survey images (before bad pixel filtering) of five galaxies detected in Hi with the MB survey at $|b| \leq 5^{\circ}$; the $I_{c}$-band image is at the top, $J$ in the middle, and $K_{s}$ at the bottom.

$\mathrm{s}^{-1}$ ), and the other galaxies may be further away or have less Hi mass and hence not be detectable with the shallow Hi survey, the cross-identifications are not always clearcut. For such cases, follow-up observations, either with Hi mapping or optical and NIR spectroscopy, are required.

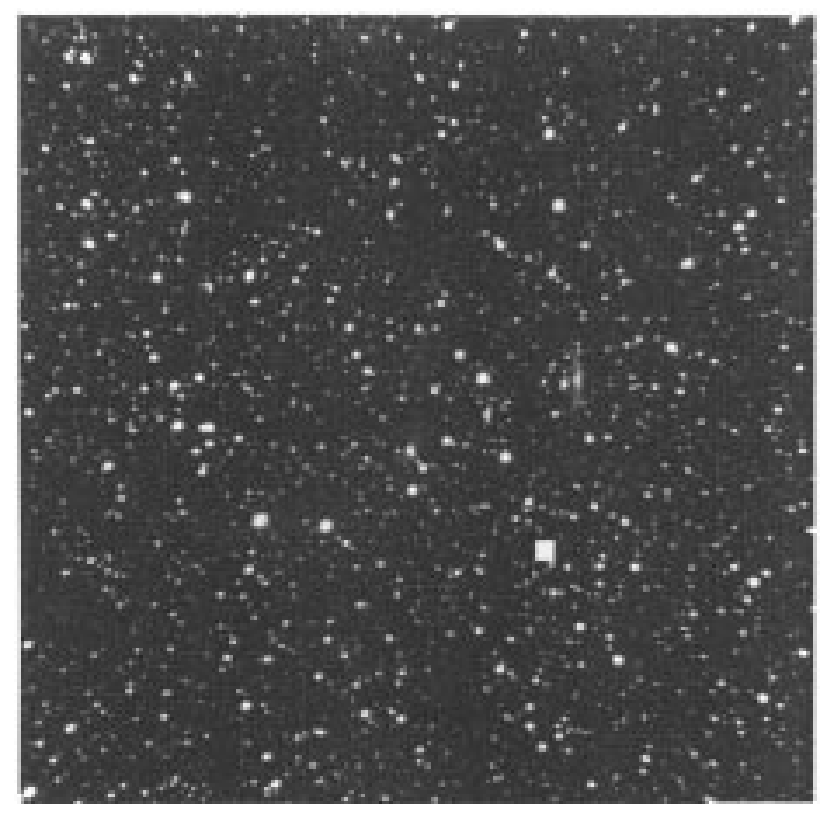

Figure 4-A $12^{\prime} \times 12^{\prime}$ DENIS $I_{c}$-band image of an MB detection (white rectangle) and four galaxy candidates (with an uncertainty radius of $\sim 4^{\prime}$ ).

\section{Photometry}

We have used a preliminary galaxy pipeline (Mamon et al. 1997b, 1998), based upon the SExtractor package (Bertin \& Arnouts 1996) to obtain $I_{c}, J$ and $K_{s}$ Kron photometry from the DENIS images at the location of MB galaxies. Although many of the galaxies have a considerable number of stars superimposed on their images, magnitudes derived from this automated algorithm agree well with independent measurements.

Figure 5 shows the dependence of the colour $J-K$ on foreground extinction $A_{B}$. Included are data from the low-latitude $\left(\ell=325^{\circ}, \quad b=-7^{\circ}\right)$, rich cluster Abell 3627 (cf. Paper II), where the foreground extinction is less than $2^{\mathrm{m}}$. The group of MB galaxies with $2^{\mathrm{m}}<A_{B}<5^{\mathrm{m}}$ shows a similar scatter but is offset in colour with respect to the galaxies in A3627. Also, the galaxy at $A_{B}=11^{\mathrm{m}} .8$ is considerably redder than all the other galaxies.

In Paper I we discussed the NIR colour-colour diagram $I-J$ versus $J-K$ of galaxies in the A3627 cluster in detail. We found that the extinctioncorrected colours of the cluster galaxies do match the colours of galaxies in unobscured high latitude regions (Mamon et al. 1997b, 1998), suggesting that our preliminary photometry is reasonably accurate. The shift in colour can be fully explained by the foreground extinction. Hence, the NIR colours of obscured galaxies (in particular of elliptical galaxies) provide an independent way of mapping 
the extinction in the ZOA (see also Mamon et al. 1997a) and calibrating the DIRBE maps at low Galactic latitudes (see also Mamon et al. 1997a).

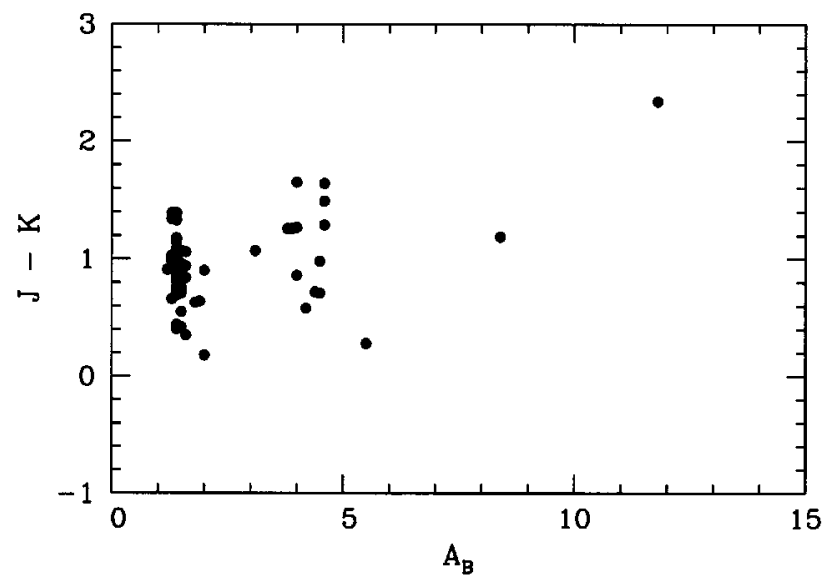

Figure 5-NIR colour as a dependence on foreground extinction.

\section{Conclusions and Future Plans}

A combination of the deep optical, systematic blind Hi and NIR surveys illustrates the potential of a multi-wavelength approach for extragalactic largescale studies behind the Milky Way. It furthermore allows the mapping of the peculiar velocity field across the ZOA through the NIR Tully-Fisher relation.

At intermediate latitudes and extinction $\left(5^{\circ}<\right.$ $\left.|b|<10^{\circ}, 1^{\mathrm{m}} \lesssim A_{B} \lesssim 4-5^{\mathrm{m}}\right)$, optical surveys remain superior for identifying galaxies due to their fainter magnitude limits. However, the NIR luminosities and colours will prove invaluable in analysing the optical survey data and their distribution in redshift space, and in the final merging of these data with existing sky surveys. Despite the high extinction and the star crowding at these latitudes, $I_{c}, J$ and $K_{s}$ photometry from the survey data can be successfully performed and lead, for instance, to preliminary $I_{c}^{0}$, $J^{0}$ and $K_{s}^{0}$ galaxy luminosity functions in A3627 (Paper II).

At low latitudes and high extinction $\left(|b|<5^{\circ}\right.$ and $A_{B} \gtrsim 4-5^{\mathrm{m}}$ ), the search for 'invisible' obscured galaxies on existing DENIS images shows that NIR surveys can trace galaxies down to about $|b| \simeq 1.5$. The $J$-band was found to be optimal for identifying galaxies up to $A_{B} \simeq 7^{\mathrm{m}}$. NIR surveys can hence further reduce the width of the $\mathrm{ZOA}$. They are furthermore the only tool that permits the mapping of early-type galaxies - tracers of density peaks - at high extinction.

The blind Hi survey uncovers spiral galaxies independent of foreground extinction. For a significant fraction, a DENIS counterpart has been found. These MB-ZOA data cover the Galactic latitude range $|b|<5^{\circ}$. We will complement this area with pointed Hi observations of optically identified spiral galaxies for intermediate latitudes $\left(5^{\circ}<|b|<10^{\circ}\right)$. About 300 spiral galaxies have already been detected (Kraan-Korteweg, Woudt \& Henning 1997).

In the near future, we plan on obtaining deep $K_{s^{-}}$ band follow-up observations of the deep and complete Hi survey in the southern ZOA (cf. Juraszek 1999, this issue p. 38). This will allow us (1) to study the effects of extinction on the extent and magnitudes of disks in the NIR, (2) to obtain a representative sample of galaxies across the ZOA for the application of the $K_{s}$-band Tully-Fisher relation, and (3) to study the morphology of the detected galaxies. The third will also be useful to understand the nature of the large-scale structures revealed with the blind Hi survey, in particular as this galaxy sample can readily be merged with the southern sky Hi survey (Kilborn 1999, this issue p. 8).

\section{Acknowledgments}

We thank Jean Borsenberger for providing bias subtracted, flat fielded DENIS images, Emmanuel Bertin for supplying recent updates of his SExtractor software package, and Eric Copet for providing software to display Figures 3 and 4. We also thank Sebastian Juraszek, Elaine Sadler and Patricia Henning for supplying us with their results of the MB survey of the ZOA.

\section{References}

Bertin, E., \& Arnouts, S. 1996, A\&AS 117, 398

Cardelli, J. A., Clayton, G. C., \& Mathis, J. S. 1989, ApJ, 345,245

Epchtein, N. 1997, in The Impact of Large Scale Near-Infrared Surveys, ed. F. Garzon et al. (Dordrecht: Kluwer), p. 15 Epchtein, N., et al. 1997, Messenger, 87, 27

Henning, P. A., Staveley-Smith, L., Kraan-Korteweg, R. C., \& Sadler, E. 1999, PASA, 16, 35

Juraszek, S. 1999, PASA, 16, 38

Kilborn, V. A., Webster, R. L., \& Staveley-Smith, L. 1999, PASA, 16, 8

Kraan-Korteweg, R. C., \& Woudt, P. A. 1994, in Unveiling Large-Scale Structures Behind the Milky Way, ASP Conf. Ser. 67, ed. C. Balkowski \& R. C. Kraan-Korteweg (San Francisco: ASP), p. 89

Kraan-Korteweg, R. C., \& Woudt, P. A. 1999, PASA, 16, 53

Kraan-Korteweg, R. C., Loan, A. J., Burton, W. B., et al. 1994, Nature, 372, 77

Kraan-Korteweg, R. C., Fairall, A. P., \& Balkowski, C. 1995, A\&A, 297, 617

Kraan-Korteweg, R. C., Woudt, P. A., Cayatte, V., et al. 1996, Nature, 379, 519

Kraan-Korteweg, R. C., Woudt, P. A., \& Henning, P. A. 1997, PASA, 14, 15

Kraan-Korteweg, R. C., Schröder, A., Mamon, G., \& Ruphy, S. 1998, Third Euroconference on the Impact of Near-Infrared Surveys on Galactic and Extragalactic Astronomy, ed. N. Epchtein (Dordrecht: Kluwer), p. 209

Lauberts, A. 1982, The ESO/Uppsala Survey of the ESO (B) Atlas (Garching: ESO)

Mamon, G. A. 1994, in Unveiling Large-Scale Structures Behind the Milky Way, ASP Conf. Ser. 67, ed. C. 
Balkowski \& R. C. Kraan-Korteweg (San Francisco: ASP), p. 53

Mamon, G. A., Banchet, V., Tricottet, M., \& Katz, D. $1997 a$, in The Impact of Large Scale Near-Infrared Surveys, ed. F. Garzon et al. (Dordrecht: Kluwer), p. 239

Mamon, G. A., Tricottet, M., Bonin, W., \& Banchet, V. 1997b, 27th Moriond Astrophysics Meeting on Extragalactic Astronomy in the Infrared, ed. G. A. Mamon et al. (Paris: Frontières), p. 369

Mamon, G. A., Borsenberger, J., Tricottet, M., \& Banchet, V. 1998, Third Euroconference on the Impact of NearInfrared Surveys on Galactic and Extragalactic Astronomy, ed. N. Epchtein (Dordrecht: Kluwer), p. 177

Schlegel, D. J., Finkbeiner, D. P., \& Davis, M. 1998, ApJ, 500,525
Schröder, A., Kraan-Korteweg, R. C., Mamon, G., \& Ruphy, S. 1997, 27th Moriond Astrophysics Meeting on Extragalactic Astronomy in the Infrared, ed. G. A. Mamon et al. (Paris: Frontières), p. 381

Sigad, Y., Eldar, A., Dekel, A., et al. 1998, ApJ, 495, 516 Skrutskie, M. F., et al. 1997, in The Impact of Large Scale Near-Infrared Surveys, ed. F. Garzon et al. (Dordrecht: Kluwer), p. 25

Staveley-Smith, L. 1997, PASA, 14, 111

Woudt, P. A. 1998, PhD thesis, Univ. of Cape Town

Woudt, P. A., Kraan-Korteweg, R. C., Fairall, A. P., et al. 1998, A\&A, 338, 8 